\title{
Quantum simulation of open quantum systems in heavy-ion collisions
}

\author{
Wibe A. de Jong $\odot{ }^{1, *}$ Mekena Metcalf, ${ }^{1, \dagger}$ James Mulligan $\oplus^{2,3, \$}$ Mateusz Płoskoń, ${ }^{2, \S}$ \\ Felix Ringer, $2, \|$ and Xiaojun Yao $\oplus^{4, \pi}$ \\ ${ }^{1}$ Computational Research Division, Lawrence Berkeley National Laboratory, \\ Berkeley, California 94720, USA \\ ${ }^{2}$ Nuclear Science Division, Lawrence Berkeley National Laboratory, Berkeley, California 94720, USA \\ ${ }^{3}$ Physics Department, University of California, Berkeley, California 94720, USA \\ ${ }^{4}$ Center for Theoretical Physics, Massachusetts Institute of Technology, \\ Cambridge, Massachusetts 02139, USA
}

(Received 16 October 2020; accepted 13 July 2021; published 7 September 2021)

\begin{abstract}
We present a framework to simulate the dynamics of hard probes such as heavy quarks or jets in a hot, strongly coupled quark-gluon plasma (QGP) on a quantum computer. Hard probes in the QGP can be treated as open quantum systems governed in the Markovian limit by the Lindblad equation. However, due to large computational costs, most current phenomenological calculations of hard probes evolving in the QGP use semiclassical approximations of the quantum evolution. Quantum computation can mitigate these costs and offers the potential for a fully quantum treatment with exponential speed-up over classical techniques. We report a simplified demonstration of our framework on IBM Q quantum devices and apply the random identity insertion method to account for CNOT depolarization noise, in addition to measurement error mitigation. Our work demonstrates the feasibility of simulating open quantum systems on current and near-term quantum devices, which is of broad relevance to applications in nuclear physics, quantum information, and other fields.
\end{abstract}

DOI: 10.1103/PhysRevD.104.L051501

\section{INTRODUCTION}

Considerable advancements in quantum devices, such as qubit coherence times, have recently been achieved [1-4]. Together with parallel progress in quantum algorithms and executable quantum software, nontrivial quantum computations can be carried out, including hybrid quantumclassical algorithms such as the variational quantum eigensolver [5-10] and fully quantum simulations of the unitary time evolution of closed quantum systems [11,12]. In high energy and nuclear physics, a variety of quantum computing applications have emerged [13-37]. In particular, quantum simulation can be applied to study dynamics of large size systems that are in principle intractable with classical methods. To perform such simulations, quantum

\footnotetext{
*wadejong@lbl.gov †mmetcalf@lbl.gov

*james.mulligan@berkeley.edu

\$mploskon@lbl.gov

fmringer@lbl.gov

"xjyao@mit.edu
}

Published by the American Physical Society under the terms of the Creative Commons Attribution 4.0 International license. Further distribution of this work must maintain attribution to the author(s) and the published article's title, journal citation, and DOI. Funded by SCOAP. circuits compiled into single and multiqubit gates can be implemented on digital quantum computers.

Many physical systems of interest are not closed, but consist of a subsystem interacting with an environment. The dynamics of the subsystem can be formulated as an open quantum system. In the Markovian limit (in which the environment correlation time is much smaller than the subsystem relaxation time), the evolution of the subsystem is governed by a generalization of the Schrödinger equation known as the Lindblad equation [38-40], where instead of keeping track of all of the environmental degrees of freedom, one only needs to record environment correlators that are relevant for the subsystem evolution. A key challenge in extending quantum simulation to open quantum systems is that the Lindblad evolution is nonunitary. During the last decade, algorithms have been developed to overcome this issue, most of which couple the subsystem with auxiliary qubits (whose dimension can be significantly smaller than that of the environment) such that the whole system evolves unitarily [41-47]. More recently, simulations of open quantum systems have been carried out on real quantum devices, but without error mitigation [48].

In this paper, we focus on the application of quantum simulations of open quantum systems to relativistic heavy-ion collisions (HICs). Experiments at the Relativistic Heavy Ion Collider (RHIC) and the Large Hadron Collider (LHC) create a hot $(T \approx 150-500 \mathrm{MeV})$, short-lived 
$(t \approx 10 \mathrm{fm} / c)$ quark-gluon plasma (QGP) [49-56]. The QGP is a deconfined phase of QCD matter believed to have existed shortly after the big bang [57]. The properties of the QGP can be investigated using jets or heavy quarks [58-62] that involve energy scales much larger than the QGP temperature ("hard probes").

The evolution of hard probes in the QGP can be treated as an open system evolving in a hot medium. A fully fieldtheoretical description of hard probes in the medium is challenging, and typically various approximations are made. Most studies employ semiclassical Boltzmann or Fokker-Planck (equivalent to Langevin) equations [63-70]; semiclassical transport equations are leading order terms in the gradient expansion of the Wigner transformed Lindblad equation [71,72]. Recently, several studies have applied Lindblad equations directly to investigate quarkonia [73-80] and jets $[81,82]$, which are valid if the subsystem and environment are weakly coupled. It is expected that as the size of the subsystem increases (such as the jet radiation phase space, or the number of heavy quarks $[83,84]$ in the subsystem), solving Lindblad equations would challenge the limits of classical computation. Quantum computing offers a possibility to remove the constraint on the subsystem size and go beyond the approximations made in semiclassical approaches. Moreover, quantum simulation may provide a solution to the notoriously difficult sign problem in classical lattice QCD calculations of real time observables [14,85-87] (the same problem can also appear in open QCD systems).

In this paper, we outline a formulation of the evolution of hard probes in the QGP as a Lindblad equation and explore how simulations on noisy intermediate scale quantum (NISQ [13]) devices can be used to advance theoretical studies of hard probes in the QGP. Using a quantum algorithm for simulating the Lindblad equation, we study a toy model on IBM Q simulators and quantum devices, and implement error mitigation for measurement and twoqubit gate noise. We demonstrate that quantum algorithms simulating simple Lindblad evolution are tractable on current and near-term devices, in terms of available number of qubits, gate depth, and error rates.

\section{OPEN QUANTUM SYSTEM FORMULATION OF HARD PROBES IN HEAVY-ION COLLISIONS}

The Hamiltonian of the full system consisting of the hard probe (subsystem) and the QGP (environment) can be written as

$$
\begin{gathered}
H=H_{S}+H_{E}+H_{I} \\
H_{S}=H_{S 0}+H_{S 1} .
\end{gathered}
$$

Here $H_{S}, H_{E}$ and $H_{I}$ are the Hamiltonians of the subsystem, the environment and their interaction, respectively. A schematic diagram of the setup is shown in Fig. 1.

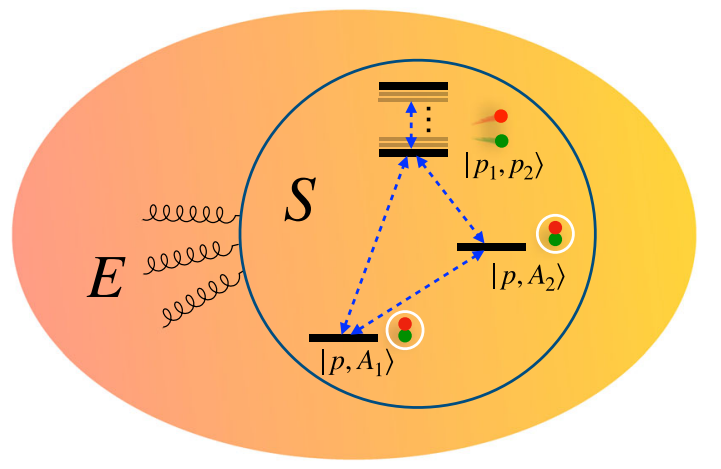

FIG. 1. A schematic illustration of a multilevel open quantum system $S$ interacting with a thermal environment $E$. The levels in $S$ can represent for example: (1) heavy quark-antiquark $(Q \bar{Q})$ bound states $\left|\boldsymbol{p}, A_{i}\right\rangle$ with center-of-mass momentum $\boldsymbol{p}$ and quantum numbers $A_{i}$, and (2) unbound $Q \bar{Q}$ pairs $\left|\boldsymbol{p}_{1}, \boldsymbol{p}_{2}\right\rangle$ with momenta $\boldsymbol{p}_{1}, \boldsymbol{p}_{2}$. For jets the levels of $S$ can represent multiparton states labeled by momenta $\left|p_{1}, \ldots, p_{n}\right\rangle$.

We further split $H_{S}$ into the free $H_{S 0}$ and the interacting part of the subsystem $H_{S 1}$. In quantum field theories, Hamiltonians are functionals of fields, which require discretization in position space [16]. Here, instead of simulating the dynamics of fields, we focus on simulating the dynamics of particle states, which is valid for hard probes. If we use multiparticle states $\left|p_{1}, A_{1}\right\rangle \otimes \cdots \otimes$ $\left|p_{n}, A_{n}\right\rangle$ as the basis where $p_{i}$ is the four-momentum, $A_{i}$ represents all discrete quantum numbers, and $i=1,2, \ldots, n$, then both $H_{S 0}$ and $H_{S 1}$ are matrices and $H_{S 0}$ is diagonal. Note that $H_{S 1}$ is different from $H_{I}$ : the former is the interaction within the subsystem itself and independent of the environment, while the latter represents the interaction between the subsystem and the environment. For example, for jets in HICs, $H_{S 1}$ can be collinear radiation of collinear particles while $H_{I}$ can describe the Glauber exchange between collinear particles (subsystem) and soft fields from the QGP environment [81].

The total density matrix of the subsystem and the environment evolves under the von Neumann equation. In the interaction picture, this is given by

$$
\frac{\mathrm{d}}{\mathrm{d} t} \rho^{(\mathrm{int})}(t)=-i\left[H_{I}^{(\mathrm{int})}(t), \rho^{(\mathrm{int})}(t)\right]
$$

The operators are defined by

$$
\begin{gathered}
\rho^{(\mathrm{int})}(t) \equiv e^{i\left(H_{S 0}+H_{E}\right) t} \rho(t) e^{-i\left(H_{S 0}+H_{E}\right) t} \\
H_{S 1}^{(\mathrm{int})}(t) \equiv e^{i H_{S 0} t} H_{S 1} e^{-i H_{S 0} t} \\
H_{I}^{(\mathrm{int})}(t) \equiv e^{i\left(H_{S 0}+H_{E}\right) t} H_{I} e^{-i\left(H_{S 0}+H_{E}\right) t} .
\end{gathered}
$$

The interaction picture used here is special: it is the standard interaction picture for the subsystem, but it is 
the Heisenberg picture for the environment. We will drop the superscript (int) from now on for simplicity but the reader should be reminded that we use the interaction picture throughout. We assume that the initial density matrix factorizes and the environment density matrix is a thermal state, ${ }^{1}$

$$
\begin{gathered}
\rho(0)=\rho_{S}(0) \otimes \rho_{E} \\
\rho_{E}=\frac{e^{-\beta H_{E}}}{\operatorname{Tr}\left(e^{-\beta H_{E}}\right)},
\end{gathered}
$$

where $\beta=1 / T$ is the inverse of the QGP temperature.

After the environment is traced out, the reduced evolution of the subsystem density matrix is generally timeirreversible and nonunitary. If the coupling between the subsystem and the environment is weak, the reduced evolution equation can be cast as a Markovian Lindblad equation [38-40],

$$
\begin{aligned}
\frac{\mathrm{d}}{\mathrm{d} t} \rho_{S}(t)= & -i\left[H_{S 1}(t)+H_{L}, \rho_{S}(t)\right] \\
& +\sum_{j=1}^{m}\left(L_{j} \rho_{S}(t) L_{j}^{\dagger}-\frac{1}{2}\left\{L_{j}^{\dagger} L_{j}, \rho_{S}(t)\right\}\right),
\end{aligned}
$$

where $H_{L}$ denotes a thermal correction to $H_{S}$ generated by loop effects of $H_{I}$, and the $L_{j}$ are called Lindblad operators, whose explicit expressions will be given for a toy model below. In general, if the dimension of the subsystem is $d$ [i.e., $\rho_{S}(t)$ is a $d \times d$ matrix], the number of independent Lindblad operators is $m=d^{2}-1$. When evaluating the Lindblad operators, an environment correlator of the form $\operatorname{Tr}_{E}\left[O_{E}\left(t_{1}\right) O_{E}\left(t_{2}\right) \rho_{E}\right]$ is needed as input, where the $O_{E}$ 's are some environment operators. This correlator can be evaluated perturbatively in thermal field theory if the environment is weakly-coupled. But the construction of the Lindblad equation only requires $H_{I}$ to be weak. In general $H_{E}$ itself can be strongly coupled, in which case the correlator has to be computed nonperturbatively using lattice QCD [99-103] or the AdS/CFT correspondence [104-108]. For the nonperturbative computation, one needs to formulate the theory such that the relevant correlator is gauge invariant, where effective field theory can be used. A concrete construction of gauge invariant correlators for quarkonium transport can be found in Refs. [72,109].

\section{QUANTUM ALGORITHM}

We will apply a quantum algorithm based on the Stinespring dilation theorem, see for example

\footnotetext{
${ }^{1}$ The backreaction of the QGP medium to jet energy loss [88-97], which may further modify jet observables is beyond the scope of our considerations here. For a recent review, see Ref. [98].
}

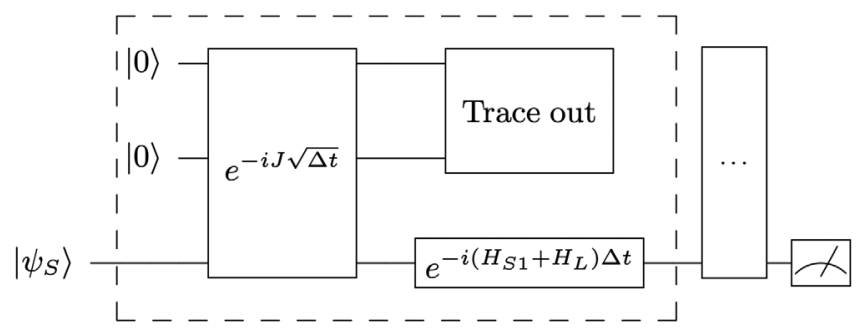

FIG. 2. Quantum algorithm to simulate Lindblad evolution based on the Stinespring dilation theorem. The portion enclosed by the dashed line corresponds to one "cycle" of time $\Delta t$. Each cycle is repeated multiple times as indicated by the ellipsis in the box on the right. The measurement of the subsystem is performed at the end.

Refs. $[44,110]$, to simulate the Lindblad equation. The algorithm in terms of the evolution operators $J$, defined below, and $H_{S}$, is illustrated in Fig. 2. The algorithm couples the subsystem with auxiliary qubits, which are traced out after each time step $\Delta t$. The dimension of the auxiliary register is $m+1$ and the number of qubits needed in practice for the register is ceil $\left(\log _{2}(m+1)\right) \equiv\left\lceil 2 \log _{2} d\right\rceil$. Together with the number of qubits required to record the subsystem state, the total number of qubits needed is $\left\lceil 3 \log _{2} d\right\rceil$. We use $\left\{|0\rangle_{a},|1\rangle_{a} \cdots,|m\rangle_{a}\right\}$ to label the basis of the auxiliary register, indicated by the subscript $a$.

We assume the initial state $\rho_{S}(0)=\left|\psi_{S}(0)\right\rangle\left\langle\psi_{S}(0)\right|$ is a pure state. ${ }^{2}$ At the beginning of each cycle at time $t$, the total density matrix of the subsystem and the auxiliary is set to be a $(m+1) \times(m+1)$ block matrix,

$\rho(t)=|0\rangle_{a}\left\langle\left. 0\right|_{a} \otimes \rho_{S}(t)=\left(\begin{array}{cccc}\rho_{S}(t) & 0 & \ldots & 0 \\ 0 & 0 & \ldots & 0 \\ \vdots & \vdots & \ddots & \vdots \\ 0 & 0 & \ldots & 0\end{array}\right)\right.$.

The $J$-operator is also a $(m+1) \times(m+1)$ block matrix,

$$
J=\left(\begin{array}{cccc}
0 & L_{1}^{\dagger} & \ldots & L_{m}^{\dagger} \\
L_{1} & 0 & \ldots & 0 \\
\vdots & \vdots & \ddots & \vdots \\
L_{m} & 0 & \ldots & 0
\end{array}\right),
$$

where each block is a $d \times d$ matrix. One can show that the circuit in Fig. 2 reproduces (9) when $\Delta t \rightarrow 0$. To simulate the evolution from 0 to $t$, the size of the time steps is $\Delta t=t / N_{\text {cycle }}$ where $N_{\text {cycle }}$ is the number of cycles; see Fig. 2.

\footnotetext{
${ }^{2}$ If it is a mixed state, then we decompose it into a linear superposition of pure states. We just need to apply the circuit to each pure state and take the linear superposition in the end.
} 


\section{TOY MODEL AND SIMULATION ON IBM Q}

Simulating real jets and heavy quarks on quantum devices requires a large number of fault-tolerant qubits. As a proof of concept, we consider the following toy model that includes qualitative features of hard probes:

$$
\begin{gathered}
H_{S}=H_{S 0}=-\frac{\Delta E}{2} Z \\
H_{E}=\int \mathrm{d}^{3} x\left[\frac{1}{2} \Pi^{2}+\frac{1}{2}(\nabla \phi)^{2}+\frac{1}{2} m^{2} \phi^{2}+\frac{1}{4 !} \lambda \phi^{4}\right] \\
H_{I}=g X \otimes \phi(x=0),
\end{gathered}
$$

where we use $X, Z$ to denote the single qubit Pauli gates (Pauli matrices). The subsystem Hamiltonian $H_{S}$ is a two level system with energy difference $\Delta E$. The two levels can correspond to the bound and unbound state of a heavy quark-antiquark pair, exchanging energy with QGP. The environment $H_{E}$ is a $3+1 D$ scalar field theory, that together with (7) mimics the thermal QGP. Here $\Pi$ is the canonical momentum conjugate to $\phi$. The extension to gauge theories requires a gauge invariant formulation of the environment correlator as mentioned earlier. The environment correlator can be calculated nonperturbatively to all orders in $\lambda$. Here for simplicity, we set $m=\lambda=0$. Nonvanishing $m$ and $\lambda$ lead to different coefficients of the Lindblad operators but do not alter the quantum algorithm. The interaction strength $g$ between the subsystem and the environment is unitless. In the Markovian limit, two Lindblad operators $j=0,1$ are relevant,

$$
L_{j}=\frac{\sqrt{\Gamma_{j}}}{2}\left(X-(-1)^{j} i Y\right)
$$

where $\Gamma_{0}=g^{2} \Delta E n_{B}(\Delta E) /(2 \pi), \quad \Gamma_{1}=g^{2} \Delta E /(2 \pi)+\Gamma_{0}$ and $n_{B}(\Delta E)=1 /(\exp (\beta \Delta E)-1)$ is the Bose-Einstein distribution. We will neglect $H_{L}$ in this paper. For our numerical studies, we use a unit system where all quantities are counted in units of $T$, the temperature of the medium. We initialize the state as $\rho_{S}(t=0)=|0\rangle\langle 0|$ and choose $\Delta E=1(T)$.

The result for this toy model obtained from the IBM Q qiskit simulator [111] is shown in Fig. 3. We measure $P_{0}(t) \equiv\left\langle 0\left|\rho_{S}(t)\right| 0\right\rangle$, which can be interpreted as the time-dependent nuclear modification factor. Each time point corresponds to an independent quantum circuit, where the measurement is performed only at the end, as shown in Fig. 2. The results of the quantum algorithm with $N_{\text {cycle }}=100$ are shown for different values of the coupling $g$. They are consistent with the results obtained with a fourth order Runge-Kutta method that solves Eq. (9) classically. This agreement demonstrates that the circuit successfully solves the Lindblad equation. As expected, the

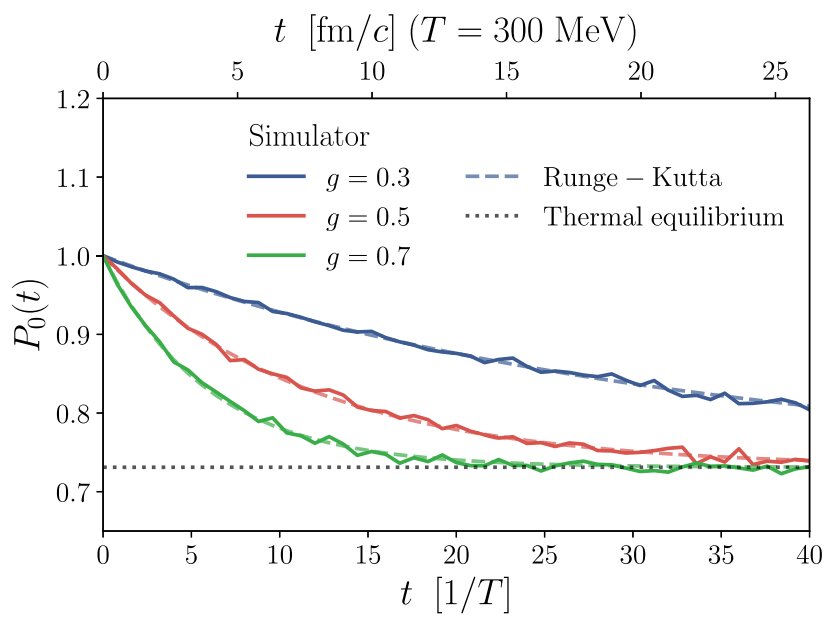

FIG. 3. Simulation of the quantum circuit with $N_{\text {cycle }}=100$ for various system-environment couplings, along with numerical solution using a fourth order Runge-Kutta method. The upper time axis corresponds to a medium with a temperature of $T=300 \mathrm{MeV}$. Each time point in the simulator result consists of 80192 shots (runs).

strength of the coupling $g$ controls the rate of approaching thermalization.

In order to run the circuit on a quantum device, we select $N_{\text {cycle }}=1$ in order to achieve a sufficiently small circuit depth. Modern quantum software packages are available to compile quantum circuits that approximate general unitary operators with minimal error and optimal depth $[10,112-114]$. We synthesize a circuit for the $e^{-i J \sqrt{\Delta t}}$ operator in terms of single qubit and CNOT gates using the qsearch compiler [114]. The compiler yields circuits with 70 gates on average, including approximately ten CNOTs per cycle; an example circuit for one cycle is shown in the Supplemental Material [115].

The results obtained from IBM Q Vigo device [116] are shown in Fig. 4. In addition to the uncorrected result, the results with readout and CNOT error mitigation are also shown. We correct the readout error using the constrained matrix inversion approach in IBM's qiskit-ignis package. The response matrix can be found in the Supplemental Material [115]. We also correct for CNOT noise using a leading order zero-noise extrapolation based on the recently developed resource efficient random identity insertion method (RIIM) [117]. This procedure corrects for depolarization noise using a set of additional $(\mathrm{CNOT})^{2}$ identity insertions, at the expense of amplifying statistical noise. Each data point corresponds to five evenly spaced time points that are averaged together. Each time point is calculated from the average of 49152 shots (runs). We observe that the error mitigation is more important at small values of $t$. Similar results were reproduced on the IBM Q Valencia and Santiago devices [118,119].

Overall, we observe good agreement of the results from the quantum device with the results from the simulator for 


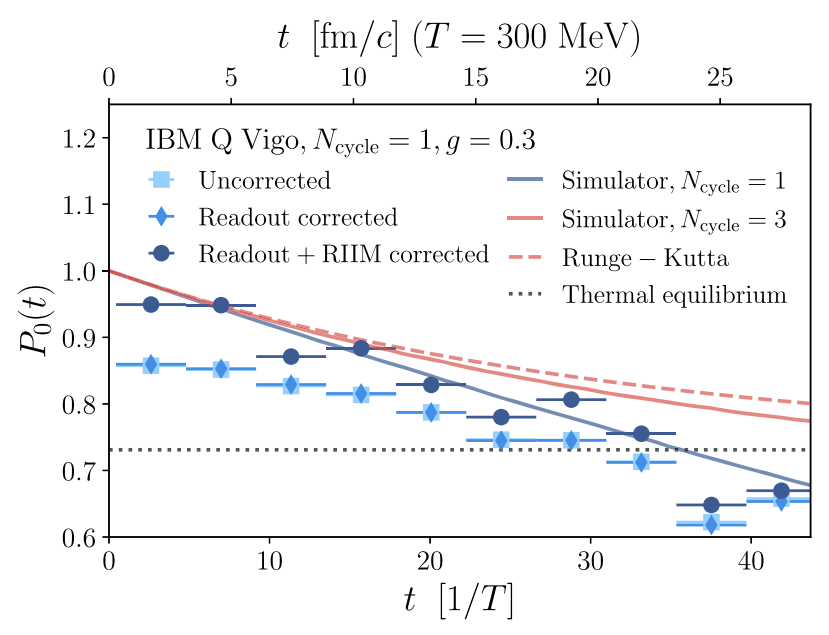

FIG. 4. Results from the IBM Q Vigo device including different error mitigations compared to results from the qiskit simulator for $N_{\text {cycle }}=1$ and $N_{\text {cycle }}=3$ and the Runge-Kutta method. Higher values of $N_{\text {cycle }}$ quickly converge to the result using the Runge-Kutta method. Each time point in the simulator result consists of 800192 shots (runs).

$N_{\text {cycle }}=1$ after the error mitigation is applied. The choice of $N_{\text {cycle }}=1$ is seen to be a reasonable approximation for sufficiently small $t$. Moreover, a modest increase to $N_{\text {cycle }}=3$, as shown by the simulator in Fig. 4, yields a considerably improved convergence, which is promising for near-term applications. These results demonstrate that the simulation of open quantum system dynamics relevant for HICs should be feasible on current and near-term quantum devices.

\section{CONCLUSIONS AND OUTLOOK}

We performed simulations of open quantum systems using quantum devices from IBM Q. In particular, we focused on simulating the nonunitary evolution of a subsystem governed by the Lindblad equation. We demonstrated that digital quantum simulations with a few qubits and a circuit depth of $\sim 70$ gate operations with $\sim 10$ CNOT gates are feasible on current quantum devices. We used the qsearch compiler to construct the quantum circuit, and implemented two-qubit gate error mitigation using zero noise extrapolation with the random identity insertion method (RIIM), in addition to readout error mitigation. Simulating open quantum systems is of great importance for theoretical studies of hard probes in heavy-ion collisions. The open quantum system formulation allows one to go beyond semiclassical transport calculations currently used in most phenomenological studies. Future calculations, using a time dependent environment density matrix may allow one to explore a broad range of physical models by varying medium properties such as the initial temperature, microscopic structure, or the probe-medium coupling. Open quantum systems are also relevant for various other systems in nuclear and high-energy physics such as studies of cold nuclear matter effects at the future ElectronIon Collider [120], the resummation of large logarithms relevant for jet physics [121-124] and studies of the color glass condensate $[125,126]$.

\section{ACKNOWLEDGMENTS}

We thank Christian Bauer, Volker Koch, Ben Nachman, Long-Gang Pang, Krishna Rajagopal, Phiala Shanahan, Rishi Sharma, Ramona Vogt, Xin-Nian Wang, and Feng Yuan for helpful discussions. We acknowledge use of IBM Q for this work. The views expressed are those of the authors and do not reflect the official policy or position of IBM or the IBM Q team. J. M., M. P. are supported by the U.S. Department of Energy, Office of Science, Office of Nuclear Physics, under the Contract No. DE-AC0205CH11231. F. R. is supported by LDRD funding from Berkeley Lab provided by the U.S. Department of Energy under Contract No. DE-AC02-05CH11231. X. Y. is supported by the U.S. Department of Energy, Office of Science, Office of Nuclear Physics under grant Contract No. DE-SC0011090. M. M. and W. D. J. were supported by the U.S. Department of Energy, Office of Science, Office of Advanced Scientific Computing Research Accelerated Research in Quantum Computing program under Contract No. DE-AC02-05CH11231. This research used resources of the Oak Ridge Leadership Computing Facility, which is a User Facility supported by U.S. Department of Energy, Office of Science, under Contract No. DE-AC0500OR22725.
[1] M. Devoret and R. Schoelkopf, Superconducting circuits for quantum information: An outlook, Science 339, 1169 (2013).

[2] M. Kjaergaard, M. E. Schwartz, J. Braumller, P. Krantz, J. I.-J. Wang, S. Gustavsson, and W. D. Oliver, Superconducting qubits: Current state of play, Annu. Rev. Condens. Matter Phys. 11, 369 (2020).
[3] C. D. Bruzewicz, J. Chiaverini, R. McConnell, and J. M. Sage, Trapped-ion quantum computing: Progress and challenges, Appl. Phys. Rev. 6, 021314 (2019).

[4] F. Arute, K. Arya, R. Babbush, D. Bacon, J. C. Bardin, R. Barends, R. Biswas, S. Boixo, F. G. S. L. Brandao, and D. A. Buell, Quantum supremacy using a programmable superconducting processor, Nature (London) 574, 505 (2019). 
[5] J. R. McClean, J. Romero, R. Babbush, and A. AspuruGuzik, The theory of variational hybrid quantum-classical algorithms, New J. Phys. 18, 023023 (2016).

[6] A. Peruzzo, J. McClean, P. Shadbolt, M.-H. Yung, X.-Q. Zhou, P. J. Love, A. Aspuru-Guzik, and J. L. OBrien, A variational eigenvalue solver on a photonic quantum processor, Nat. Commun. 5, 4213 (2014).

[7] A. Kandala, A. Mezzacapo, K. Temme, M. Takita, M. Brink, J. M. Chow, and J. M. Gambetta, Hardwareefficient variational quantum eigensolver for small molecules and quantum magnets, Nature (London) 549, 242 (2017).

[8] Google AI Quantum and Collaborators, Hartree-fock on a superconducting qubit quantum computer, Science 369, 1084 (2020).

[9] J. I. Colless, V. V. Ramasesh, D. Dahlen, M. S. Blok, M. E. Kimchi-Schwartz, J. R. McClean, J. Carter, W. A. de Jong, and I. Siddiqi, Computation of Molecular Spectra on a Quantum Processor with an Error-Resilient Algorithm, Phys. Rev. X 8, 011021 (2018).

[10] F. T. Chong, D. Franklin, and M. Martonosi, Programming languages and compiler design for realistic quantum hardware, Nature (London) 549, 180 (2017).

[11] L. Bassman, K. Liu, A. Krishnamoorthy, T. Linker, Y. Geng, D. Shebib, S. Fukushima, F. Shimojo, R. K. Kalia, A. Nakano, and P. Vashishta, Towards simulation of the dynamics of materials on quantum computers, Phys. Rev. B 101, 184305 (2020).

[12] A. Smith, M. S. Kim, F. Pollmann, and J. Knolle, Simulating quantum many-body dynamics on a current digital quantum computer, npj Quantum Inf. 5, 106 (2019).

[13] J. Preskill, Quantum computing in the nisq era and beyond, Quantum 2, 79 (2018).

[14] S. P. Jordan, K. S. Lee, and J. Preskill, Quantum algorithms for quantum field theories, Science 336, 1130 (2012).

[15] D. B. Kaplan, N. Klco, and A. Roggero, Ground states via spectral combing on a quantum computer, arXiv: 1709.08250 .

[16] J. Preskill, Simulating quantum field theory with a quantum computer, Proc. Sci., LATTICE2018 (2018) 024.

[17] N. Klco and M. J. Savage, Digitization of scalar fields for quantum computing, Phys. Rev. A 99, 052335 (2019).

[18] E. Dumitrescu, A. McCaskey, G. Hagen, G. Jansen, T. Morris, T. Papenbrock, R. Pooser, D. Dean, and P. Lougovski, Cloud Quantum Computing of an Atomic Nucleus, Phys. Rev. Lett. 120, 210501 (2018).

[19] N. Klco, E. Dumitrescu, A. McCaskey, T. Morris, R. Pooser, M. Sanz, E. Solano, P. Lougovski, and M. Savage, Quantum-classical computation of Schwinger model dynamics using quantum computers, Phys. Rev. A 98, 032331 (2018).

[20] C. C. Chang, A. Gambhir, T. S. Humble, and S. Sota, Quantum annealing for systems of polynomial equations, Sci. Rep. 9, 10258 (2019).

[21] A. Roggero, A. C. Li, J. Carlson, R. Gupta, and G. N. Perdue, Quantum computing for neutrino-nucleus scattering, Phys. Rev. D 101, 074038 (2020).

[22] N. Klco, J. R. Stryker, and M. J. Savage, SU(2) nonAbelian gauge field theory in one dimension on digital quantum computers, Phys. Rev. D 101, 074512 (2020).
[23] A. Roggero and A. Baroni, Short-depth circuits for efficient expectation value estimation, Phys. Rev. A 101, 022328 (2020).

[24] I. C. Clot et al., Opportunities for nuclear physics \& quantum information science, in Intersections between Nuclear Physics and Quantum Information, edited by I. C. Clot and M. R. Dietrich (2019), https://www.osti .gov/biblio/1523421-opportunities-nuclear-physics-ampquantum-information-science.

[25] C. W. Bauer, W. A. De Jong, B. Nachman, and D. Provasoli, A Quantum Algorithm for High Energy Physics Simulations, Phys. Rev. Lett. 126, 187603 (2021).

[26] N. Mueller, A. Tarasov, and R. Venugopalan, Deeply inelastic scattering structure functions on a hybrid quantum computer, Phys. Rev. D 102, 016007 (2020).

[27] A. Y. Wei, P. Naik, A. W. Harrow, and J. Thaler, Quantum algorithms for jet clustering, Phys. Rev. D 101, 094015 (2020).

[28] E. T. Holland, K. A. Wendt, K. Kravvaris, X. Wu, W. Erich Ormand, J. L. DuBois, S. Quaglioni, and F. Pederiva, Optimal control for the quantum simulation of nuclear dynamics, Phys. Rev. A 101, 062307 (2020).

[29] A. Avkhadiev, P. Shanahan, and R. Young, Accelerating Lattice Quantum Field Theory Calculations via Interpolator Optimization Using Noisy Intermediate-Scale Quantum Computing, Phys. Rev. Lett. 124, 080501 (2020).

[30] A. F. Shaw, P. Lougovski, J. R. Stryker, and N. Wiebe, Quantum algorithms for simulating the lattice Schwinger Model, Quantum 4, 306 (2020).

[31] J. Liu and Y. Xin, Quantum simulation of quantum field theories as quantum chemistry, J. High Energy Phys. 12 (2020) 011.

[32] M. Kreshchuk, W. M. Kirby, G. Goldstein, H. Beauchemin, and P. J. Love, Quantum simulation of quantum field theory in the light-front formulation, arXiv:2002.04016.

[33] D. E. Kharzeev and Y. Kikuchi, Real-time chiral dynamics from a digital quantum simulation, Phys. Rev. Research 2 , 023342 (2020).

[34] N. Klco and M. J. Savage, Fixed-point quantum circuits for quantum field theories, Phys. Rev. A 102, 052422 (2020).

[35] O. Di Matteo, A. McCoy, P. Gysbers, T. Miyagi, R. Woloshyn, and P. Navrátil, Improving Hamiltonian encodings with the Gray code, Phys. Rev. A 103, 042405 (2021).

[36] Z. Davoudi, I. Raychowdhury, and A. Shaw, Search for efficient formulations for Hamiltonian simulation of nonabelian lattice gauge theories, arXiv:2009.11802.

[37] K. Bepari, S. Malik, M. Spannowsky, and S. Williams, Towards a quantum computing algorithm for helicity amplitudes and parton showers, Phys. Rev. D 103, 076020 (2021).

[38] A. Kossakowski, On quantum statistical mechanics of non-hamiltonian systems, Rep. Math. Phys. 3, 247 (1972).

[39] G. Lindblad, On the generators of quantum dynamical semigroups, Commun. Math. Phys. 48, 119 (1976).

[40] V. Gorini, A. Frigerio, M. Verri, A. Kossakowski, and E. Sudarshan, Properties of quantum Markovian master equations, Rep. Math. Phys. 13, 149 (1978).

[41] H. Wang, S. Ashhab, and F. Nori, Quantum algorithm for simulating the dynamics of an open quantum system, Phys. Rev. A 83, 062317 (2011). 
[42] R. Sweke, I. Sinayskiy, D. Bernard, and F. Petruccione, Universal simulation of markovian open quantum systems, Phys. Rev. A 91, 062308 (2015).

[43] S.-J. Wei, D. Ruan, and G.-L. Long, Duality quantum algorithm efficiently simulates open quantum systems, Sci. Rep. 6, 30727 (2016).

[44] R. Cleve and C. Wang, Efficient Quantum Algorithms for Simulating Lindblad Evolution, in 44th International Colloquium on Automata, Languages, and Programming (ICALP 2017).

[45] A. Chenu, M. Beau, J. Cao, and A. del Campo, Quantum Simulation of Generic Many-Body Open System Dynamics Using Classical Noise, Phys. Rev. Lett. 118, 140403 (2017).

[46] H.-Y. Su and Y. Li, Quantum algorithm for the simulation of open-system dynamics and thermalization, Phys. Rev. A 101, 012328 (2020).

[47] M. Metcalf, J. E. Moussa, W. A. de Jong, and M. Sarovar, Engineered thermalization and cooling of quantum manybody systems, Phys. Rev. Res. 2, 023214 (2020).

[48] Z. Hu, R. Xia, and S. Kais, A quantum algorithm for evolving open quantum dynamics on quantum computing devices, Sci. Rep. 10, 3301 (2020).

[49] J. D. Bjorken, Highly relativistic nucleus-nucleus collisions: The central rapidity region, Phys. Rev. D 27, 140 (1983).

[50] I. Arsene et al. (BRAHMS Collaboration), Quark gluon plasma and color glass condensate at RHIC? The Perspective from the BRAHMS experiment, Nucl. Phys. A757, 1 (2005).

[51] K. Adcox et al. (PHENIX Collaboration), Formation of dense partonic matter in relativistic nucleus-nucleus collisions at RHIC: Experimental evaluation by the PHENIX Collaboration, Nucl. Phys. A757, 184 (2005).

[52] B. Back et al. (PHOBOS Collaboration), The PHOBOS perspective on discoveries at RHIC, Nucl. Phys. A757, 28 (2005).

[53] J. Adams et al. (STAR Collaboration), Experimental and theoretical challenges in the search for the quark gluon plasma: The STAR Collaboration's critical assessment of the evidence from RHIC collisions, Nucl. Phys. A757, 102 (2005).

[54] B. Müller, J. Schukraft, and B. Wysłouch, First Results from $\mathrm{Pb}+\mathrm{Pb}$ Collisions at the LHC, Annu. Rev. Nucl. Part. Sci. 62, 361 (2012).

[55] P. Braun-Munzinger, V. Koch, T. Schäfer, and J. Stachel, Properties of hot and dense matter from relativistic heavy ion collisions, Phys. Rep. 621, 76 (2016).

[56] W. Busza, K. Rajagopal, and W. van der Schee, Heavy Ion Collisions: The Big Picture, and the Big Questions, Annu. Rev. Nucl. Part. Sci. 68, 339 (2018).

[57] S. Weinberg, The First Three Minutes. A Modern View of the Origin of the Universe (Bantam Books, 1977), ISBN: 9780465024377.

[58] A. Adare et al. (PHENIX Collaboration), Heavy Quark Production in $p+p$ and Energy Loss and Flow of Heavy Quarks in $\mathrm{Au}+\mathrm{Au}$ Collisions at $\sqrt{s_{N N}}=200 \mathrm{GeV}$, Phys. Rev. C 84, 044905 (2011).

[59] A. M. Sirunyan et al. (CMS Collaboration), Measurement of prompt and nonprompt charmonium suppression in
$\mathrm{PbPb}$ collisions at $5.02 \mathrm{TeV}$, Eur. Phys. J. C 78, 509 (2018).

[60] L. Adamczyk et al. (STAR Collaboration), Measurements of jet quenching with semi-inclusive hadron + jet distributions in $\mathrm{Au}+\mathrm{Au}$ collisions at $\sqrt{s_{N N}}=200 \mathrm{GeV}$, Phys. Rev. C 96, 024905 (2017).

[61] S. Acharya et al. (ALICE Collaboration), Measurements of inclusive jet spectra in $\mathrm{pp}$ and central $\mathrm{Pb}-\mathrm{Pb}$ collisions at $\sqrt{s_{\mathrm{NN}}}=5.02 \mathrm{TeV}$, Phys. Rev. C 101, 034911 (2020).

[62] M. Aaboud et al. (ATLAS Collaboration), Measurement of the nuclear modification factor for inclusive jets in $\mathrm{Pb}+\mathrm{Pb}$ collisions at $\sqrt{s_{\mathrm{NN}}}=5.02 \mathrm{TeV}$ with the ATLAS detector, Phys. Lett. B 790, 108 (2019).

[63] P. Gossiaux and J. Aichelin, Towards an understanding of the RHIC single electron data, Phys. Rev. C 78, 014904 (2008).

[64] B. Schenke, C. Gale, and S. Jeon, MARTINI: An Event generator for relativistic heavy-ion collisions, Phys. Rev. C 80, 054913 (2009).

[65] X.-N. Wang and Y. Zhu, Medium Modification of $\gamma$-Jets in High-Energy Heavy-Ion Collisions, Phys. Rev. Lett. 111, 062301 (2013).

[66] S. Cao, T. Luo, G.-Y. Qin, and X.-N. Wang, Linearized Boltzmann transport model for jet propagation in the quark-gluon plasma: Heavy quark evolution, Phys. Rev. C 94, 014909 (2016).

[67] S. Cao, G.-Y. Qin, and S. A. Bass, Energy loss, hadronization and hadronic interactions of heavy flavors in relativistic heavy-ion collisions, Phys. Rev. C 92, 024907 (2015).

[68] X. Du, R. Rapp, and M. He, Color screening and regeneration of bottomonia in high-energy heavy-ion collisions, Phys. Rev. C 96, 054901 (2017).

[69] W. Ke, Y. Xu, and S. A. Bass, Linearized BoltzmannLangevin model for heavy quark transport in hot and dense QCD matter, Phys. Rev. C 98, 064901 (2018).

[70] X. Yao, W. Ke, Y. Xu, S. A. Bass, and B. Müller, Coupled Boltzmann transport equations of heavy quarks and quarkonia in quark-gluon plasma, J. High Energy Phys. 01 (2021) 046.

[71] J.-P. Blaizot and M. A. Escobedo, Quantum and classical dynamics of heavy quarks in a quark-gluon plasma, J. High Energy Phys. 06 (2018) 034.

[72] X. Yao and T. Mehen, Quarkonium semiclassical transport in quark-gluon plasma: Factorization and quantum correction, J. High Energy Phys. 02 (2021) 062.

[73] C. Young and K. Dusling, Quarkonium above deconfinement as an open quantum system, Phys. Rev. C 87, 065206 (2013).

[74] Y. Akamatsu and A. Rothkopf, Stochastic potential and quantum decoherence of heavy quarkonium in the quarkgluon plasma, Phys. Rev. D 85, 105011 (2012).

[75] P. B. Gossiaux and R. Katz, Upsilon suppression in the Schrdinger-Langevin approach, Nucl. Phys. A956, 737 (2016).

[76] N. Brambilla, M. A. Escobedo, J. Soto, and A. Vairo, Heavy quarkonium suppression in a fireball, Phys. Rev. D 97, 074009 (2018).

[77] X. Yao and T. Mehen, Quarkonium in-medium transport equation derived from first principles, Phys. Rev. D 99, 096028 (2019). 
[78] T. Miura, Y. Akamatsu, M. Asakawa, and A. Rothkopf, Quantum Brownian motion of a heavy quark pair in the quark-gluon plasma, Phys. Rev. D 101, 034011 (2020).

[79] R. Sharma and A. Tiwari, Quantum evolution of quarkonia with correlated and uncorrelated noise, Phys. Rev. D 101, 074004 (2020).

[80] N. Brambilla, M. A. Escobedo, M. Strickland, A. Vairo, P. Vander Griend, and J.H. Weber, Bottomonium suppression in an open quantum system using the quantum trajectories method, J. High Energy Phys. 05 (2021) 136.

[81] V. Vaidya and X. Yao, Transverse momentum broadening of a jet in quark-gluon plasma: An open quantum system EFT, J. High Energy Phys. 10 (2020) 024.

[82] V. Vaidya, Effective field theory for jet substructure in heavy ion collisions, arXiv:2010.00028.

[83] A. Andronic, P. Braun-Munzinger, K. Redlich, and J. Stachel, Evidence for charmonium generation at the phase boundary in ultra-relativistic nuclear collisions, Phys. Lett. B 652, 259 (2007).

[84] A. Abada et al. (FCC Collaboration), FCC physics opportunities: Future circular collider conceptual design report Volume 1, Eur. Phys. J. C 79, 474 (2019).

[85] S. P. Jordan, K. S. Lee, and J. Preskill, Quantum computation of scattering in scalar quantum field theories, Quantum Inf. Comput. 14, 1014 (2014).

[86] E. A. Martinez, C. A. Muschik, P. Schindler, D. Nigg, A. Erhard, M. Heyl, P. Hauke, M. Dalmonte, T. Monz, P. Zoller et al., Real-time dynamics of lattice gauge theories with a few-qubit quantum computer, Nature (London) 534, 516 (2016).

[87] J. F. Haase, L. Dellantonio, A. Celi, D. Paulson, A. Kan, K. Jansen, and C. A. Muschik, A resource efficient approach for quantum and classical simulations of gauge theories in particle physics, Quantum 5, 393 (2021).

[88] J. Casalderrey-Solana, E. Shuryak, and D. Teaney, Conical flow induced by quenched QCD jets, J. Phys. Conf. Ser. 27, 22 (2005).

[89] J. Ruppert and B. Mller, Waking the colored plasma, Phys. Lett. B 618, 123 (2005).

[90] A. Chaudhuri and U. Heinz, Effect of Jet Quenching on the Hydrodynamical Evolution of QGP, Phys. Rev. Lett. 97, 062301 (2006).

[91] G.-Y. Qin, A. Majumder, H. Song, and U. Heinz, Energy and Momentum Deposited Into a QCD Medium by a Jet Shower, Phys. Rev. Lett. 103, 152303 (2009).

[92] B. Betz, J. Noronha, G. Torrieri, M. Gyulassy, and D. H. Rischke, Universal Flow-Driven Conical Emission in Ultrarelativistic Heavy-Ion Collisions, Phys. Rev. Lett. 105, 222301 (2010).

[93] S. Floerchinger and K. C. Zapp, Hydrodynamics and jets in dialogue, Eur. Phys. J. C 74, 3189 (2014).

[94] W. Chen, S. Cao, T. Luo, L.-G. Pang, and X.-N. Wang, Effects of jet-induced medium excitation in $\gamma$-hadron correlation in A + A collisions, Phys. Lett. B 777, 86 (2018).

[95] L. Yan, S. Jeon, and C. Gale, Jet-medium interaction and conformal relativistic fluid dynamics, Phys. Rev. C 97, 034914 (2018).
[96] Y. Tachibana, C. Shen, and A. Majumder, Bulk medium evolution has considerable effects on jet observables!, arXiv:2001.08321.

[97] J. Casalderrey-Solana, J. G. Milhano, D. Pablos, K. Rajagopal, and X. Yao, Jet wake from linearized hydrodynamics, J. High Energy Phys. 05 (2021) 230.

[98] S. Cao and X.-N. Wang, Jet quenching and medium response in high-energy heavy-ion collisions: A review, Rep. Prog. Phys. 84, 024301 (2021).

[99] P. Petreczky and D. Teaney, Heavy quark diffusion from the lattice, Phys. Rev. D 73, 014508 (2006).

[100] D. Banerjee, S. Datta, R. Gavai, and P. Majumdar, Heavy quark momentum diffusion coefficient from lattice QCD, Phys. Rev. D 85, 014510 (2012).

[101] A. Majumder, Calculating the jet quenching parameter $\hat{q}$ in lattice gauge theory, Phys. Rev. C 87, 034905 (2013).

[102] A. Francis, O. Kaczmarek, M. Laine, T. Neuhaus, and H. Ohno, Nonperturbative estimate of the heavy quark momentum diffusion coefficient, Phys. Rev. D 92, 116003 (2015).

[103] N. Brambilla, V. Leino, P. Petreczky, and A. Vairo, Lattice QCD constraints on the heavy quark diffusion coefficient, Phys. Rev. D 102, 074503 (2020).

[104] H. Liu, K. Rajagopal, and U. A. Wiedemann, Calculating the Jet Quenching Parameter from AdS/CFT, Phys. Rev. Lett. 97, 182301 (2006).

[105] H. Liu, K. Rajagopal, and U. A. Wiedemann, Wilson loops in heavy ion collisions and their calculation in AdS/CFT, J. High Energy Phys. 03 (2007) 066.

[106] J. Casalderrey-Solana and D. Teaney, Heavy quark diffusion in strongly coupled $N=4$ Yang-Mills, Phys. Rev. D 74, 085012 (2006).

[107] S. Caron-Huot and G. D. Moore, Heavy quark diffusion in QCD and $N=4$ SYM at next-to-leading order, J. High Energy Phys. 02 (2008) 081.

[108] J. Casalderrey-Solana, H. Liu, D. Mateos, K. Rajagopal, and U. A. Wiedemann, Gauge/String Duality, Hot QCD and Heavy Ion Collisions (Cambridge University Press, Cambridge, England, 2014).

[109] N. Brambilla, M. A. Escobedo, A. Vairo, and P. Vander Griend, Transport coefficients from in medium quarkonium dynamics, Phys. Rev. D 100, 054025 (2019).

[110] M. A. Nielsen and I. L. Chuang, Quantum Computation and Quantum Information: 10th Anniversary Edition (Cambridge University Press, Cambridge, England, 2010).

[111] A. Héctor Abraham et al., Qiskit: An open-source framework for quantum computing, 2019.

[112] E. Younis, K. Sen, K. Yelick, and C. Iancu, Qfast: Quantum synthesis using a hierarchical continuous circuit space, arXiv:2003.04462.

[113] M. G. Davis, E. Smith, A. Tudor, K. Sen, I. Siddiqi, and C. Iancu, Heuristics for quantum compiling with a continuous gate set, arXiv:1912.02727.

[114] T. E. Davis, A. Tudor, K. Sen, I. Siddiqi, and C. Iancu, Towards depth optimal topology aware quantum circuit synthesis, in IEEE International Conference on Quantum Computing and Engineering (QCE20) (2020).

[115] See Supplemental Material at http://link.aps.org/ supplemental/10.1103/PhysRevD.104.L051501 for further details of the quantum circuit and error mitigation method. 
[116] 5-qubit backend: IBM Q team, IBM Q 5 Vigo backend specification v1.2.1 (2020) Retrieved from https:// quantum-computing.ibm.com.

[117] A. He, B. Nachman, W. A. de Jong, and C. W. Bauer, Zeronoise extrapolation for quantum-gate error mitigation with identity insertions, Phys. Rev. A 102, 012426 (2020).

[118] 5-qubit backend: IBM Q team, IBM Q 5 Valencia backend specification v1.3.1 (2020) Retrieved from https:// quantum-computing.ibm.com.

[119] 5-qubit backend: IBM Q team, IBM Q 5 Santiago backend specification v1.0.3 (2020) Retrieved from https:// quantum-computing.ibm.com.

[120] A. Accardi et al., Electron ion collider: The next QCD frontier: Understanding the glue that binds us all, Eur. Phys. J. A 52, 268 (2016).
[121] M. Dasgupta and G. Salam, Resummation of nonglobal QCD observables, Phys. Lett. B 512, 323 (2001).

[122] A. Banfi, G. Marchesini, and G. Smye, Away from jet energy flow, J. High Energy Phys. 08 (2002) 006.

[123] Z. Nagy and D. E. Soper, Parton showers with quantum interference, J. High Energy Phys. 09 (2007) 114.

[124] D. Neill, The edge of jets and subleading non-global logs, arXiv:1508.07568.

[125] N. Armesto, F. Dominguez, A. Kovner, M. Lublinsky, and V. Skokov, The color glass condensate density matrix: Lindblad evolution, entanglement entropy and Wigner functional, J. High Energy Phys. 05 (2019) 025.

[126] M. Li and A. Kovner, JIMWLK evolution, Lindblad equation and quantum-classical correspondence, J. High Energy Phys. 05 (2020) 036. 